TAO, Vol. 11, No. 4, 909-928, December 2000

\title{
Fault Detection by 2D Resistivity Inversion on a Topographic Area
}

\author{
Chieh-Hou Yang ${ }^{1, *}$, Ping-Hu Chen ${ }^{2}$ and Jin-Jun Chen ${ }^{2}$ \\ (Manuscript received 13 June 2000, in final form 11 October 2000)
}

\begin{abstract}
Direct current (DC) resistivity data acquired on rough terrain can be interpreted by using an appropriate two-dimensional (2-D) finite element inversion with the topography-incorporated technique developed by Tong and Yang (1990). This technique is computationally efficient and provides a geological interpretation that is an improvement on the standard 2-D inversion. The main feature of this scheme is that sounding data incorporated the topography into the inversion without any previous topographic correction of field data. Thus errors inherited by the inversion data from an imperfect topographic correction can be avoided, especially in an area that has complex subsurface structures.

In addition, the penetration depth of a Schlumberger four symmetric collinear electrode (4-electrode survey) array is often limited by obstacles at ground-level; especially in mountainous areas. This drawback may be overcome by using a Schlumberger three asymmetric collinear electrode (3-electrode survey) array. In order to demonstrate the advantage of using the 3-electrode survey, model studies were performed, and the results show that the DC resistivity responses obtained from the 4-electrode and 3-electrode surveys have similar features for a given spread length. However, under the same topographic constraints, the 3-electrode configuration has a more extension of a spread length, and therefore, a greater penetration depth.

To seek verification of our strategy, DC resistivity sounding was applied to the Kanchiao fault area of northern Taiwan. The results stressed fault detection in this area. A Schlumberger 3-electrode survey was carried out in the field, and the data collected were inverted to the geoelectric models by using Tong and Yang's scheme (1990). In addition, synthetic apparent resistivity pseudosections were also computed from the final inversion models. Comparison of the synthetic with the observed apparent
\end{abstract}

\footnotetext{
${ }_{1}^{1}$ Institute of Applied Geology, National Central University, Chungli, Taiwan, ROC

2 Institute of Geophysics, National Central University, Chungli, Taiwan, ROC

${ }^{*}$ Corresponding author address: Professor Chieh-Hou Yang, Institute of Applied Geology, National Central University, Chungli, Taiwan, ROC; Email: yang@app.geo.ncu.edu.tw
} 
resistivity profiles is sufficient to reveal the Kanchiao fault position.

The implications of the results obtained by combining the special electrode layout of the $\mathrm{DC}$ resistivity soundings with the previously mentioned finite element inversion technique are highlighted. The techniques used here does provide users with a guide of what to do in a topographic area. The techniques are superior to conventional techniques as applied to a complex subsurface structure.

(Key words: 2D resistivity sounding, Fault detection)

\section{INTRODUCTION}

Direct current (DC) resistivity electric exploration has long been used as an effective method for geologic mapping, mineral resource exploration, groundwater investigation, and civil engineering design (Zhdanov and Keller 1994). Interpretation of the sounding data is often analyzed by inversion techniques. Oristaglio and Worthington (1980), Tripp et al. (1984), and Tong and Yang (1990) developed 2-D inversion algorithms to estimate the resistivity distribution within the earth. The methods suggested by these authors provide a way to interpret complex subsurface structures in an electric survey. Since Tong and Yang's inversion scheme can be used for automatic 2-D inversion of resistivity data measured on an irregular ground surface directly, we adopted their strategy for converting field data in this study.

This research was primarily concerned with the DC resistivity sounding applied to geologic mapping, and payed particular attention to the fault investigation in a topographic area. Model studies have shown that the conventional 4-electrode array carried out on a rough relief area become either expensive or inadequate. For these reasons, a 3-electrode survey was used. In addition, incorporating the topography into the field data without previous topographic correction, by using Tong and Yang's inversion algorithm to process the field data, decresed the errors transfered to the final inversion results. The site chosen for the study is in northern Taiwan, and relates to an uncertain Kanchiao fault trace. The subsurface was mapped and the fault characteristics examined. The results indicate that a combination of a special field design for DC soundings, with Tong and Yang's inversion method, did improve the geologic interpretation of the complex subsurface structures in a topographic area.

\section{MODEL STUDY}

Vertical electric sounding (VES) using a collinear 4-electrode is widely used in geologic mapping, especially at places where the seismic method and other geophysical techniques are unsuitable. However, if the survey is carried out in a mountainous area, topography and/or obstacles will prevent an extended survey spread, making it very hard to get the information from greater depths. This drawback may be overcome by using a 3-electrode survey since it provides more available space for an extended survey spread, without losing the features of VES curves obtained by the conventional 4-electrode survey. Here, we will give some model- 
ing studies to demonstrate these benefits. We will start with some simple fundamental geoelectric models and then go through a more realistic structure.

\subsection{Three Horizontally Layered Model}

Figure 1a shows the synthetic VES curves for both 4-electrode (dashed line) and 3-electrode (solid line) surveys computed from a horizontal three-layer model. The top layer of the model has a resistivity of $1000 \mathrm{ohm}-\mathrm{m}$ and a thickness $15 \mathrm{~m}$, while those of the second layer are $100 \mathrm{ohm}-\mathrm{m}$ and $15 \mathrm{~m}$, and the last layer has a resistivity of $1000 \mathrm{ohm}-\mathrm{m}$. For the 3electrode survey, a remote stationary current electrode is positioned at a distance of $100 \mathrm{~m}$ from the measured point (midpoint between potential electrodes). The dashed line is a reference curve. The maximum of half-electrode spacing $(\mathrm{AB} / 2)$ for the 4-electrode survey is 1000 $\mathrm{m}$. As shown in the figure, the VES curves for both arrays do match very well, except for computations where the half-electrode spacing $(\mathrm{AB} / 2)$ is greater than $100 \mathrm{~m}$ which is the maximum allowable extension distance between the potential electrode and current electrode for a 3-electrode survey. In this case, the maximum spread for the 4-electrode array survey was $50 \mathrm{~m}$. Therefore, for a given spread length, the depth of penetration for a 3-electrode survey is greater than that for the 4-electrode. Figure $1 \mathrm{~b}$ shows the relative error of the VES responses obtained for 3- and 4-electrode surveys versus $\mathrm{AB} / 2$ shown in Fig. 1a. The average of the relative error for $\mathrm{AB} / 2$ of less than $100 \mathrm{~m}$ is $13.6 \%$, with a root mean square error (rms) of 24\%. Figure 2a has the same geoelectrical model as that shown in Fig.l a, except that the remote current electrode of the 3-electrode survey is positioned at $1000 \mathrm{~m}$ instead of $100 \mathrm{~m}$. The dashed and solid lines are the VES results for a 4- and a 3-electrode survey respectively. The average error between the two VES curves is less than $0.8 \%$ and the rms is less than $1.2 \%$ (Fig. 2b). Comparison of Figs. 1 and 2 it indicates that an increase in the distance between the remote stationary current electrode and the measured point decreases the relative error of the VES response obtained from a 3-electrode survey.

Figure $3 \mathrm{a}$ is the synthetic VES curve for a horizontal three-layered model using a 4-electrode survey; the computed sounding responses were terminated at $A B / 2=40 \mathrm{~m}$ by a presumed surface obstacle which prevented further extension of the survey spread. The VES curve has the features of a two-layered model. Figure $3 \mathrm{~b}$ shows a synthetic sounding curve obtained from the same earth model shown in Fig. 3a. The VES curve was computed by using a 3electrode survey. In this case, computed VES responses for $\mathrm{AB} / 2$ greater than $40 \mathrm{~m}$ could be obtained. The VES curve shows the characteristics of a three-layered earth model responses. The above results demonstrate the obvious advantage of using a 3-electrode survey in a rough relief surface.

\subsection{Trapezoidal Valley Model}

Figure $4 \mathrm{a}$ is a trapezoidal valley model with uniform resistivity. The base is 10 units deep and 35 units wide. A DC resistivity survey is carried out in the valley. The remote stationary current electrode for a 3-electrode survey is positioned 100 units from the center of the valley. The distance between the sounding location and foothill is marked by a capital D. Figure $4 \mathrm{~b}$ shows the VES curves computed by using a 3-electrode survey for both $D=12$ units and $D=2$ 

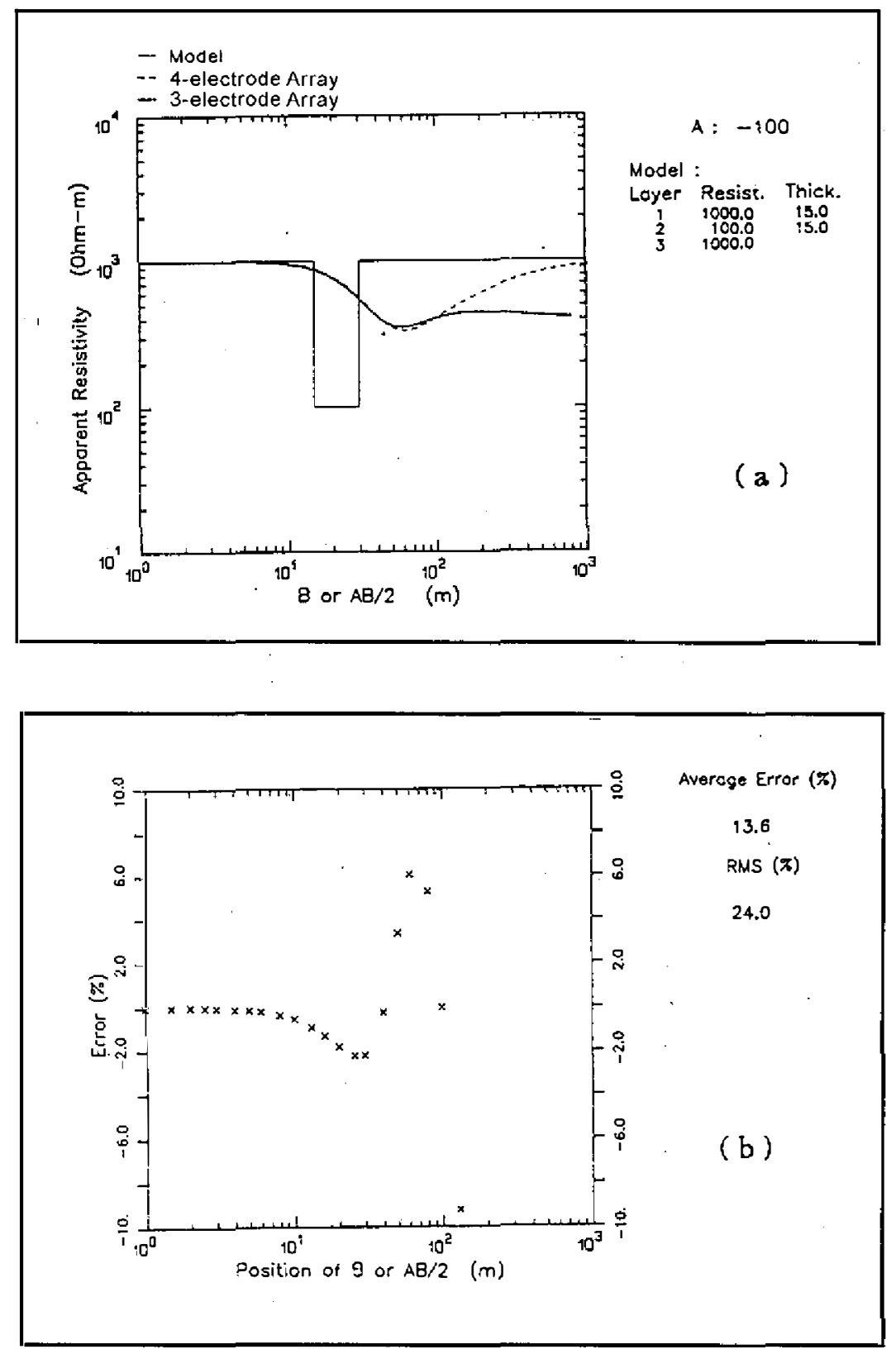

Fig. 1. (a) VES results computed from a horizontal three-layer model using both a Schlumberger 4-electrode array (dashed line) and a 3-electrode array (solid line). For the 3-electrode array, the remote current electrode is positioned $100 \mathrm{~m}$ from the sounding location. (b) The relative error and rms error of VES data for a Schlumberger 4 -electrode array with respect to a 3-electrode array. 

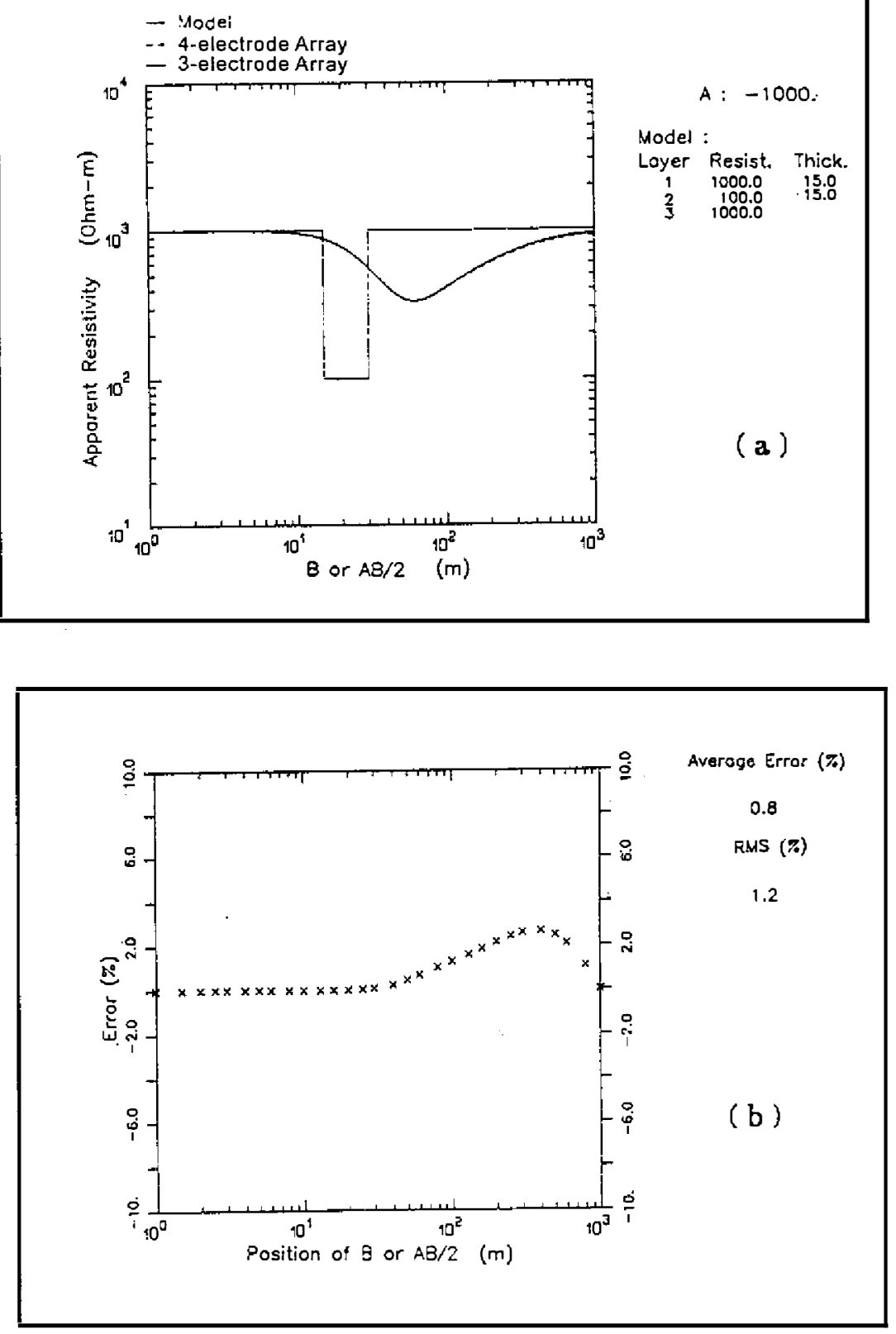

Fig. 2. (a) VES results computed from a horizontal three-layered model for both a Schlumberger 4-electrode array (dashed line) and a 3-electrode array (solid line). The remote current electrode for the 3-electrode array is positioned $1000 \mathrm{~m}$ from the sounding point. (b) The relative error and rms error of VES data for the 4-electrode array with respect to the 3electrode array. 

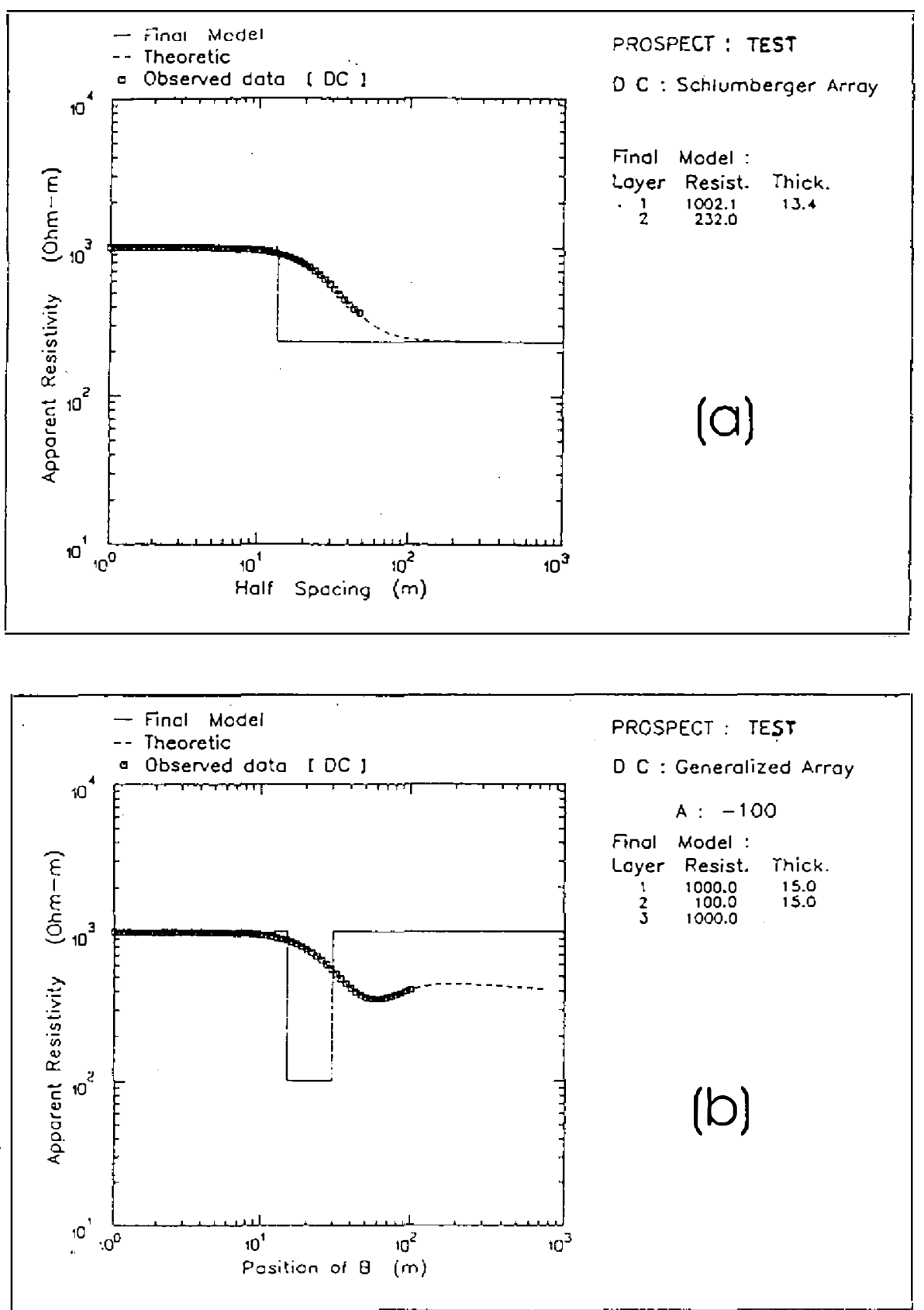

Fig. 3. (a) VES results computed from a horizontal three-layered model using a Schlumberger 4-electrode survey. Note that the sounding measurement terminated at $\mathrm{AB} / 2=40 \mathrm{~m}$ (b) VES results computed from the same geoelectric model as in (a) except that a 3-electrode survey is used. The stationary remote current electrode is positioned at $1000 \mathrm{~m}$. 


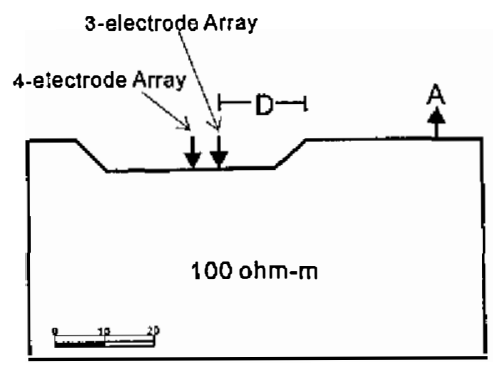

(a)

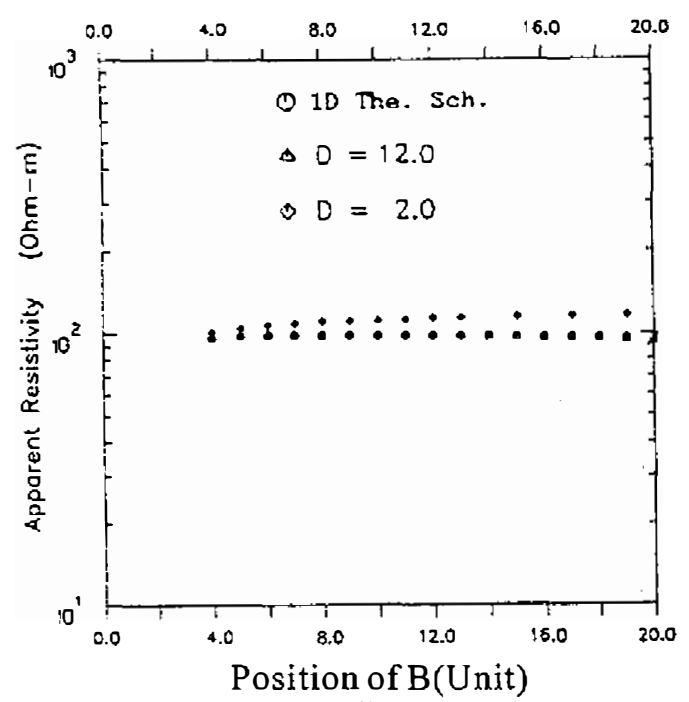

(b)

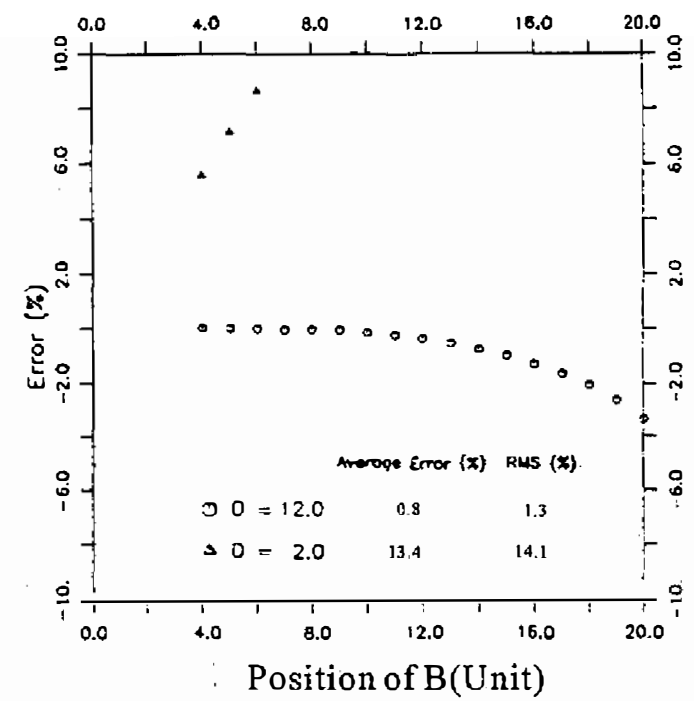

(c)
Fig. 4. (a) A trapezoidal valley model. (b) VES curves using a Schlumberger 3-electrode array with $\mathrm{D}=2$ units and $\mathrm{D}=12$ units, where $\mathrm{D}$ is the distance between the sounding location and the edge of the valley. (c) Relative errors of VES data obtained in (b) relative to the VES data obtained by a Schlumberger sounding carried out in a half-space. (d) VES data computed from the earth model shown in (a) using a 4electrode survey and 3-electrode survey. Theoretical VES data for a half-space using a Schlumberger sounding is also shown. (e) As in Fig. (d), relative errors of VES data computed from both a 4-electrode survey and a 3-electrode survey, relative to the theoretic VES data. 


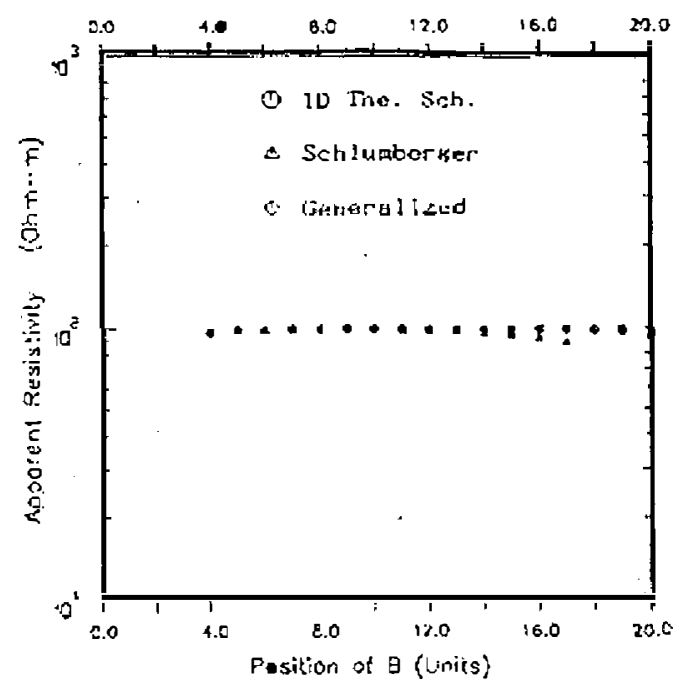

(d)

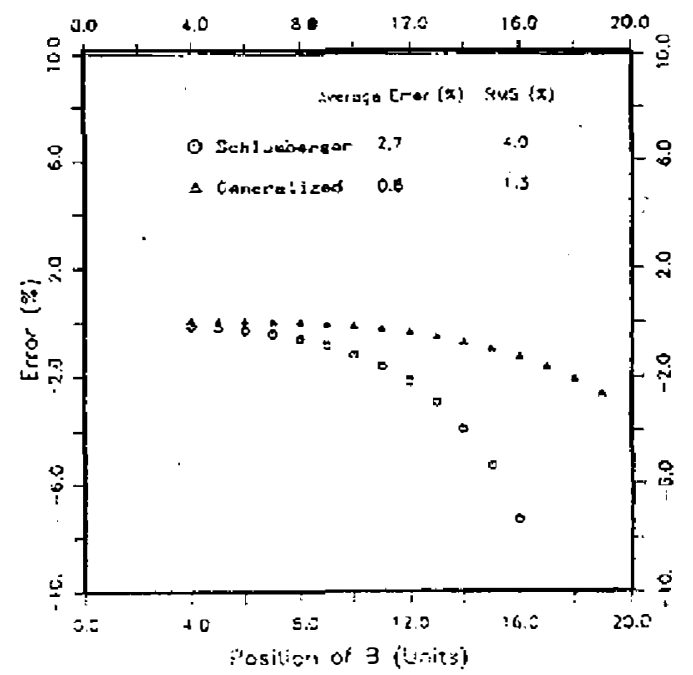

(e)

(Fig. 4. continued)

units. In these figures, the VES curve computed by a greater D matches better than the smaller $D$ with a theoretical Schlumberger VES curve (TS VES) for a half space with a flat surface. Figure $4 \mathrm{c}$ is the relative error estimated from the computed VES values either from $D=12$ units or $D=2$ units to that of TS. The error decreases as the $D$ value increases. Figure $4 \mathrm{~d}$ shows the VES curve obtained from a 4-electrode array at a location $\mathrm{D}=17$ units and also that computed from a 3-electrode array at a location $D=12$ units. Separate comparison of these two curves with the TS VES curve indicates that the synthetic VES curve for the 4-electrode survey is more sensitive to topography. Resultant error with respect to the TS VES is less for a 3electrode array than for 4-electrode survey. 
These results show that if we wish to study the deeper subsurface structure in a mountainous area, then a 3-electrode survey is far superior to a 4-electrode survey one.

\section{INVERSION METHOD}

The detailed descriptions of the finite-element method applied to the 2-D electrical and EM inversion problems have been given by Inman (1975), Vozoff and Jupp (1975), and Tong and Yang (1990), among others. Therefore, only an outline of the basic equations as they apply to the DC resistivity problem are included in this section. For more details, refer to Tong and Yang (1990).

Assume $N$ unknown model parameters $\mathbf{x}$ which are related to $M$ resistivity data $\mathbf{d}$ by a nonlinear functional $h$ defined as

$$
d_{i}=h\left(\mathbf{x}, c_{i}\right)=h_{i}(\mathbf{x}) ; i=1, \ldots \ldots, \mathrm{M} ;
$$

where $c_{i}$ denotes the electrode separations. This nonlinear equation is linearized by expansion in a Taylor series for each value of $c_{i}$ with respect to a reference model $x_{0}$. Neglecting higher order terms leads to a linear set of $\mathrm{M}$ equations in $\mathrm{N}$ unknowns.

$$
\Delta d=\underline{a} \Delta X,
$$

where

$$
\begin{aligned}
& \Delta d_{i}=h\left(x, c_{i}\right)-h\left(x_{0}, c_{i}\right) ; \\
& a_{i j}=\partial h\left(x, c_{i}\right) /\left.\partial x_{j}\right|_{x=x_{0}} ; \quad i=1, . ., M ; j=1, \ldots, N,
\end{aligned}
$$

and

$$
\Delta x=x-x_{0}
$$

The vector $\Delta d$ is the difference between the measured apparent resistivity data and the theoretical apparent resistivity of the initial model. The vector $\Delta x$ is the difference between the unknown model parameters and the initial model parameters. In our case, the model parameter is the resistivity of each block. The matrix $\underline{a}$, referred to as the Jacobian matrix, is the matrix of partial derivatives of the apparent resistivities with respect to the model parameters.

Because both the model parameters and the resistivity data values may vary over several orders of magnitude, and because they must be constrained to positive values, we use logarithmic fitting. Thus, let $\mathbf{D}=\ln \mathbf{d}, \mathbf{H}=\ln \mathbf{h}$, and $\mathbf{X}=\ln \mathbf{x}$. Using these equations and incorporating a data weighting matrix $\underline{W}$ into the Jacobian matrix and equation ( 2 ),

$$
A_{i j}=W_{i j}^{1 / 2}\left(\partial H_{i} / \partial X_{j}\right)
$$

and

$$
\Delta d=\underline{W}^{1 / 2}(D-H)=\underline{A} \Delta X
$$

The inverse procedure is to find the model parameters which minimize $\|\Delta D\|$. Since equation (4) was deduced by linearizing a nonlinear system, the inverse solution requires several iterations. At each step, equation (4) is solved for $\Delta X$ which yields a new set of model 
parameters. This procedure is repeated until an accepted minimum square residual is reached.

Gloub and Reinsch (1970) decomposed the Jacobian matrix $\underline{A}$ into its row and column eigenvectors, and the associated singular values, as

$$
\underline{A}=\underline{U} \underline{\Lambda} \underline{V}^{T}
$$

where $\underline{U}$ is an $M$ by $M$ data eigenvector matrix, $\underline{V}$ is an $N$ by $N$ solution eigenvector matrix, and $\Lambda$ is an $N$ by $N$ diagonal singular value matrix.

The parameter improvement vector $\Delta X$ is obtained by substituting $A$ from equation (5). The solution is

$$
\Delta X=\underline{V} \underline{\Lambda}^{-1} \underline{U}^{T} \Delta D
$$

The problem is that when small singular values are present, the estimate for $\Delta X$ is grossly contaminated by numerical noise. To overcome this problem, a damped $N$ by $N$ diagonal matrix $\underline{T}$ is added to equation (6); thus,

$$
\Delta X=\underline{V} \underline{T} \underline{\Lambda}^{-1} \underline{U}^{T} \Delta D
$$

The elements of $\underline{T}$ are

$$
t_{j}=k_{j}^{4} /\left(k_{j}^{4}+\mu^{4}\right),
$$

where $\mu$ is known as the relative singular value threshold and $k_{j}=\lambda_{j} / \lambda_{1} . \lambda_{j}$ is the $j$ th singular value and $\lambda_{1}$ is the maximum of the singular values. The estimate is further stabilized by initially including only the largest singular values in the estimate. As the fitting error decreases, the singular values of less importance are also included in the estimate. This is performed by initially giving $\mu$ a high value of 0.2 and then, as the fitting error decreases, permitting $\mu$ to be decreased from iteration to iteration until it reaches a minimum allowed value.

The choice of inversion model parameters in this paper is the resistivity of each block. The geometry of each block is specified and the topography is included explicitiy. The resistivity of each block was estimated in the inversion process. The final result is then the resistivity distribution under the specified irregular surface. The program developed for this application, implemented on an HP 735, incorporated the following limits: the total number of permissible blocks is 54, defined on a finite element mesh containing 169 and 20 nodes in the horizontal and vertical directions, respectively; and 113 observed resistivity points are allowed.

\section{CALCULATION OF THE JACOBIAN MATRIX}

Part of the inversion process is evaluating the partial derivatives of the apparent resistivity with respect to the model parameters. Details on partial derivative of DC responses can be cited from Tong and Yang (1990). Therefore, only an outline is included in this section. 


\subsection{Jacobian Matrix for DC Resistivity Response}

Assume that $\phi_{i 1}$ and $\phi_{i 2}$ are the electric potentials for the potential electrodes labeled "1" and "2", respectively, at the $i$ th measurement in the space domain. The measured apparent resistivity response is given by

$$
\rho_{i}=\frac{G_{i}}{I_{i}}\left|\phi_{i 1}-\phi_{i 2}\right|
$$

where $\rho_{\dot{k}}, G$, and $I$ are the apparent resistivity, geometric factor, and applied current, respectively. Besides evaluating the forward problem, an iterative inversion technique requires partial derivatives of the model response $\rho_{a}$ with respect to the model parameter $x_{j}$, so one obtains

$$
\frac{\partial \rho_{a i}}{\partial x_{j}}=\frac{G_{i}}{I_{i}}\left|\frac{\partial \phi_{i 1}}{\partial x_{j}}-\frac{\partial \phi_{i 2}}{\partial x_{j}}\right|,
$$

Since $\phi$ is the inverse Fourier transform of $\Phi$,

$$
\frac{\partial \phi}{\partial x_{j}}=F^{-1}\left(\frac{\partial \Phi}{\partial x_{j}}\right),
$$

where $F^{-1}$ denotes the inverse Fourier transform. Thus the evaluation of the partial derivative of $\Phi$ with respect to $x_{j}$ is the key point in the calculation of the Jacobian matrix.

Consider a 2-D geoelectric model with a point source on its surface. The basic concept of the finite-element solution scheme is to divide the region of interest into elements, and then, to seek a solution in terms of the electric potential at the nodes of the mesh. The electric field induced by a point source is three dimensional (3D); it is necessary to remove its variation in the strike-direction by Fourier transformation. Thus in the transform domain, we can establish the following matrix equation:

$$
\underline{K} \Phi=S,
$$

where $\underline{K}$ is an $L$ by $L$ symmetric, sparse, banded, and tridiagonally dominant matrix, which is known as the stiffness marrix. The elements of this matrix depend upon the conductivities of the model and the values of the transform variables. $L$ is the total number of nodes in the mesh. The vector $\Phi$ comprises the unknown value of electric potential at each node in the transform domain. The vector $\mathrm{S}$ contains the applied current and location of the point source.

Since $\underline{K}$ is the sum of simple element matrices, its derivative with respect to $x_{j}$ can be assembled from the appropriate derivatives of the element matrices. Thus the derivative of $\Phi$ with respect to parameter $x_{j}$ can be obtained by solving equation (13):

$$
\underline{K} \frac{\partial \Phi}{\partial x_{j}}=-\frac{\partial \underline{K}}{\partial x_{j}} \Phi
$$




\subsection{D Interpretation}

Two-dimensional (2D) interpretation of the apparent resistivity pseudosections is of course highly desirable for a full understanding of data collected with 2D coverage. The data sometimes were measured over a rough terrain surface. The joint inversion technique used here is quite similar to Tong and Yang's scheme (1990). This technique is applied to remove the errors caused by assuming a flat ground surface at the pre-2D resistivity inversion stage by incorporating topography in the inversion. Here, we give a brief description of this technique.

The first step is to feed in the apparent resistivity pseudosection data at nodes on the mesh of an element after the forward model response has been calculated. The model response is then compared with field data, and the model resistivities are automatically adjusted by using an iterative second-order Marquardt damped least-squares scheme (Tong and Yang 1990). The forward calculation is repeated. The rms value of the differences between field data and model response is calculated after each iteration. The process is repeated and the model is adjusted until an acceptable fit is achieved. The forward program used is a modified version of the finite element code for modeling 2D structures incorporating topography, presented previously by Tong and Yang (1990). The technique used involves solving a 3D potential distribution due to point sources located in a 2D half-space, often referred to as 2.5D modeling. The modeled subsurface is divided into discrete elements, in which the element sizes can vary, but their shapes are always rectangular. This means that the element can be coarsened successively towards the outer edges in order to simulate infinite extent. The resistivity distribution is defined for each element at the nodes of the mesh, and therefore can be varied arbitrarily between nodes. Topographic effects are included in the model.

The resistivity data is fed into the nodes by using the same interpolation routine as used for plotting pseudosections. Surrounding edge elements are assigned with the values of the nearest pseudosection data points. The mesh is coarsened in the $\mathrm{x}$-direction when calculating for the shortest electrode spacing. However, the mesh was designed as a trade-off between lateral coverage, sufficient depth peneration and time-memory requirements for the forward calculation. For details of the mathematical approach, refer in Tong and Yang (1990).

\section{FIELD DATA EXAMPLES}

To verify the feasibility of the method, the site located in the extended direction of a known Kanchiao fault trace in northem Taiwan was chosen. The aim of this study is to detect and locate the fault trace in the study area. Below are the geologic setting, and the field tests conducted to assess the advantage of the method.

\subsection{Geologic Setting}

The study area is located in the Kanchiao area, northern Taiwan. The whole area is covered by mountains. Stiff cliffs are widely distributed in the study area except for a small portion of low relief land along the river between Kanchiao and Wanli. The variation in altitude of the ground surface is generally greater than $600 \mathrm{~m}$. 
The Kanchiao overthrust fault is one of the largest faults in northern Taiwan, and an outcrop of the fault is located on the coast near Wanli. Figure 5 is the geological map of the Kanchiao area. As shown in the figure, the fault runs inland in a NE-SW direction with a dip of $25^{\circ} \mathrm{E}$, it is also parallel to the river Malien and then, it terminates in the Shilin. The total length of the fault is about $20 \mathrm{~km}$. The hanging wall of the Kanchiao overthrust fault is characterized by the Oligocene Wuchihshan Formation. The upper part of the Wuchihshan Formation is dominated by black shale, the rest of the formation consists of white coarse sandstone and gravel. The total thickness of the Wuchihshan Formation is about $750 \mathrm{~m}$. The foot wall is the Miocene Shihti Formation, which is dominated by white sandstone and alternations between sandstone and shale. The total thickness of this formation ranges from $300 \mathrm{~m}$ to $450 \mathrm{~m}$ (Yen and Chen 1953; Ho 1963; Hsu 1967; and Huang 1988). In spite of the existence of the Kanchiao fault, the actual position of the fault trace in the study area is still uncertain. This study tries to delineate the subsurface structure and the position of the fault by using a DC resistivity survey.

\subsection{DC profiling Soundings}

The equipment used in the survey is an ES-G1 manufactured by OYO Corporation, Japan. A total of 38 vertical electrical soundings (VES) were carried out in the study area. The length of sounding spread was often limited by the presence of high topography. Two survey profiles ( $\mathrm{RA}-\mathrm{RA}^{\prime}$ and $\mathrm{RC}-\mathrm{RC}^{\prime}$ ) were run across suspected Kanchiao fault zones. Inter-station spacing was $30 \mathrm{~m}$ to $110 \mathrm{~m}$. The survey line $\mathrm{RB}-\mathrm{RB}^{\prime}$ was run parallel to a proposed Kanchiao fault trace. In order to compare the sounding results obtained from a conventional. Schlumberger 4-electrode survey with those obtained from a Schlumberger 3-electrodes survey, the VES soundings were performed at 31 positions using both layouts. The final results show that using a 3-electrode survey can detect deeper subsurface anomalies and provide diagnostic data as well as a conventional 4-electrode survey can. Figure 6 shows a locality map of the VES locations and also the survey profiles. A maximum half current electrode spacing $(\mathrm{AB} / 2)$ of $350 \mathrm{~m}$ was used at 3-electrode survey. The data collected at a pseudodepth greater than $150 \mathrm{~m}$ (not shown in RA - RA' and RC- $\mathrm{RC}^{\prime}$ pseudosections) are used to constrain the inversion models.

\subsection{Interpretation}

A Wenner array with four equi-spaced collinear electrodes was carried out in each outcrop to set up a resistivity spectrum of the lithology in the study area. Table 1 is the resistivity spectrum obtained, and demonstrates that the resistivity of the Wuchihshan Formation is generally less than that of the Shihti Formation. However, the resistivities for both formations are comparable if both samples are wet.

In order to find a proper initial model for starting a 2D inversion of the field data, a 2D model representative profile is constructed on the basis of the 1D interpretation. Forward modeling and field data inversion are repeated until the model parameters are not varying significantly or all the observations are matched closely enough, then a geoelectric section can be 


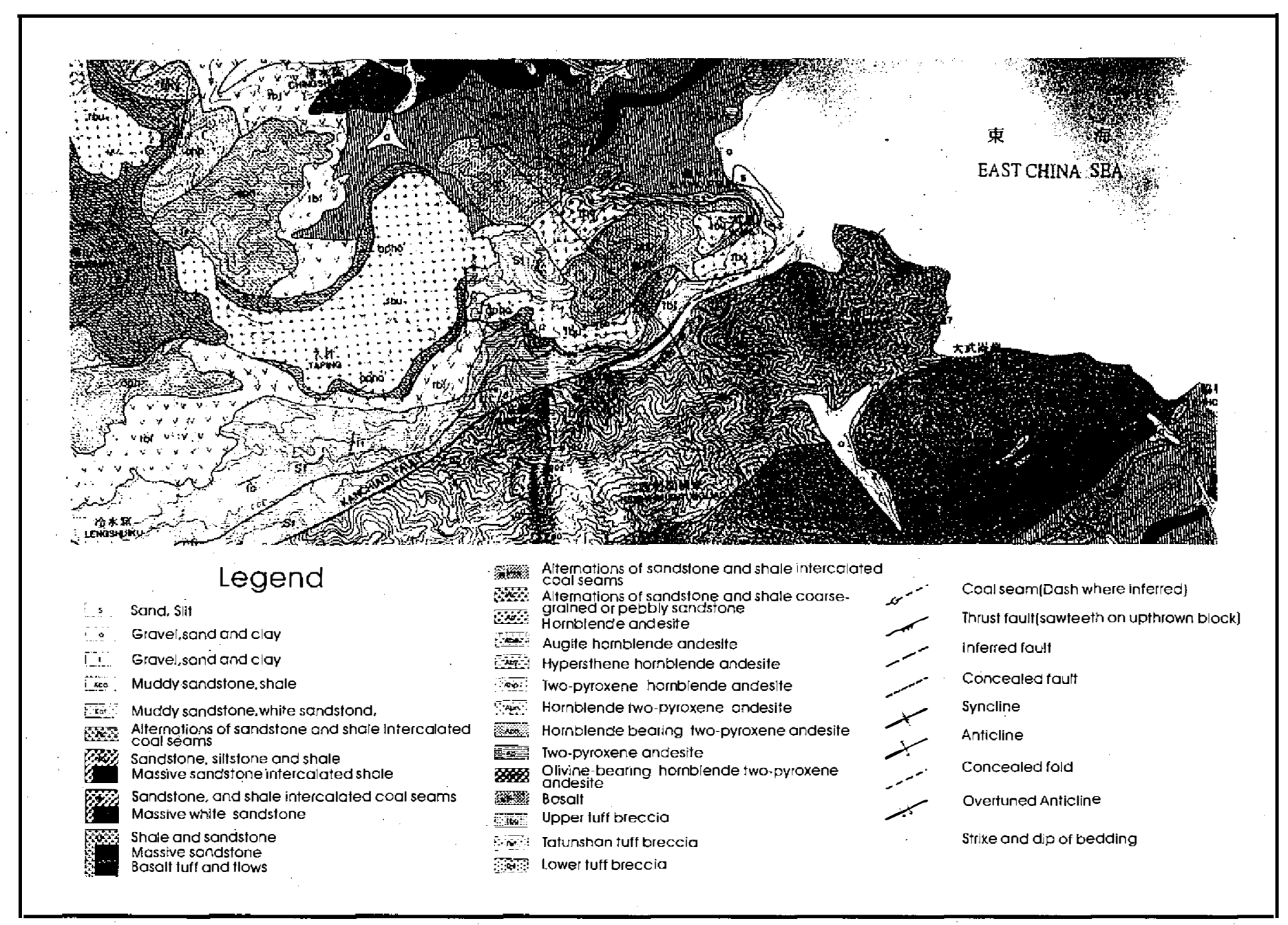

Fig. 5. The geologic map of the study area. 
Table 1. Resistivity spectrum in study area.

\begin{tabular}{|c|c|c|c|}
\hline Formation & Lithology & Dry or Wet & $\begin{array}{l}\text { Resistivity } \\
\text { (ohm-m) }\end{array}$ \\
\hline Taliao & medium size yellow sandstone & dry & 57 \\
\hline Taliao & weathering shale & dry & 75 \\
\hline$?$ & weathering laterite & dry & 424,350 \\
\hline Shihti & weathering laterite & wet & 510 \\
\hline Shihti & gray fine sandstone & dry & 74 \\
\hline $\begin{array}{l}\text { Wuchihshan } \\
\text { Shihti }\end{array}$ & $\begin{array}{l}\text { coarse quartz sandstone fine } \\
\text { sandstone }\end{array}$ & dry & $\begin{array}{l}558 \\
358 \\
\end{array}$ \\
\hline $\begin{array}{l}\text { lower } \\
\text { Wuchihshan }\end{array}$ & sandstone & dry & 353 \\
\hline Wuchihshan & \begin{tabular}{|l} 
fine sandstone \\
black silt sandstone \\
coarse quartz sandstone
\end{tabular} & dry & $\begin{array}{l}446 \\
106 \\
543 \\
\end{array}$ \\
\hline $\begin{array}{l}\text { upper } \\
\text { Wuchihshan }\end{array}$ & thick yellow fine sandstone & wet & 125 \\
\hline $\begin{array}{l}\text { upper } \\
\text { Wuchihshan }\end{array}$ & alterations of silt sandstone and shale & wet & 78 \\
\hline $\begin{array}{l}\text { lower } \\
\text { Wuchihshan }\end{array}$ & $\begin{array}{l}\text { thick coarse white sandstone } \\
\text { thick coarse gray black sandstone }\end{array}$ & dry & $\begin{array}{l}72 \\
88 \\
\end{array}$ \\
\hline $\begin{array}{l}\text { lower } \\
\text { Wuchihshan }\end{array}$ & thick coarse white sandstone & dry & 129 \\
\hline $\begin{array}{l}\text { lower } \\
\text { Wuchihshan }\end{array}$ & thick coarse white sandstone & dry & 150 \\
\hline $\begin{array}{l}\text { lower } \\
\text { Wuchihshan }\end{array}$ & thick fine gray sandstone & very dry & 155 \\
\hline Shihti & fine gray sandstone & wet & 550 \\
\hline Wuchihshan & $\begin{array}{l}\text { thick light-yellow medium quartz } \\
\text { sandstone }\end{array}$ & very dry & 283 \\
\hline Shihti & thick fine sandstone & wet & 237 \\
\hline Shihti & fine white-gray sandstone & dry & 605 \\
\hline Shihti & thin gray silt sandstone & wet & 54 \\
\hline Shihti & gray layered silt sandstone & dry & 64 \\
\hline $\begin{array}{l}\text { lower } \\
\text { Wuchihshan }\end{array}$ & coarse white quartz sandstone & dry & 1100 \\
\hline
\end{tabular}




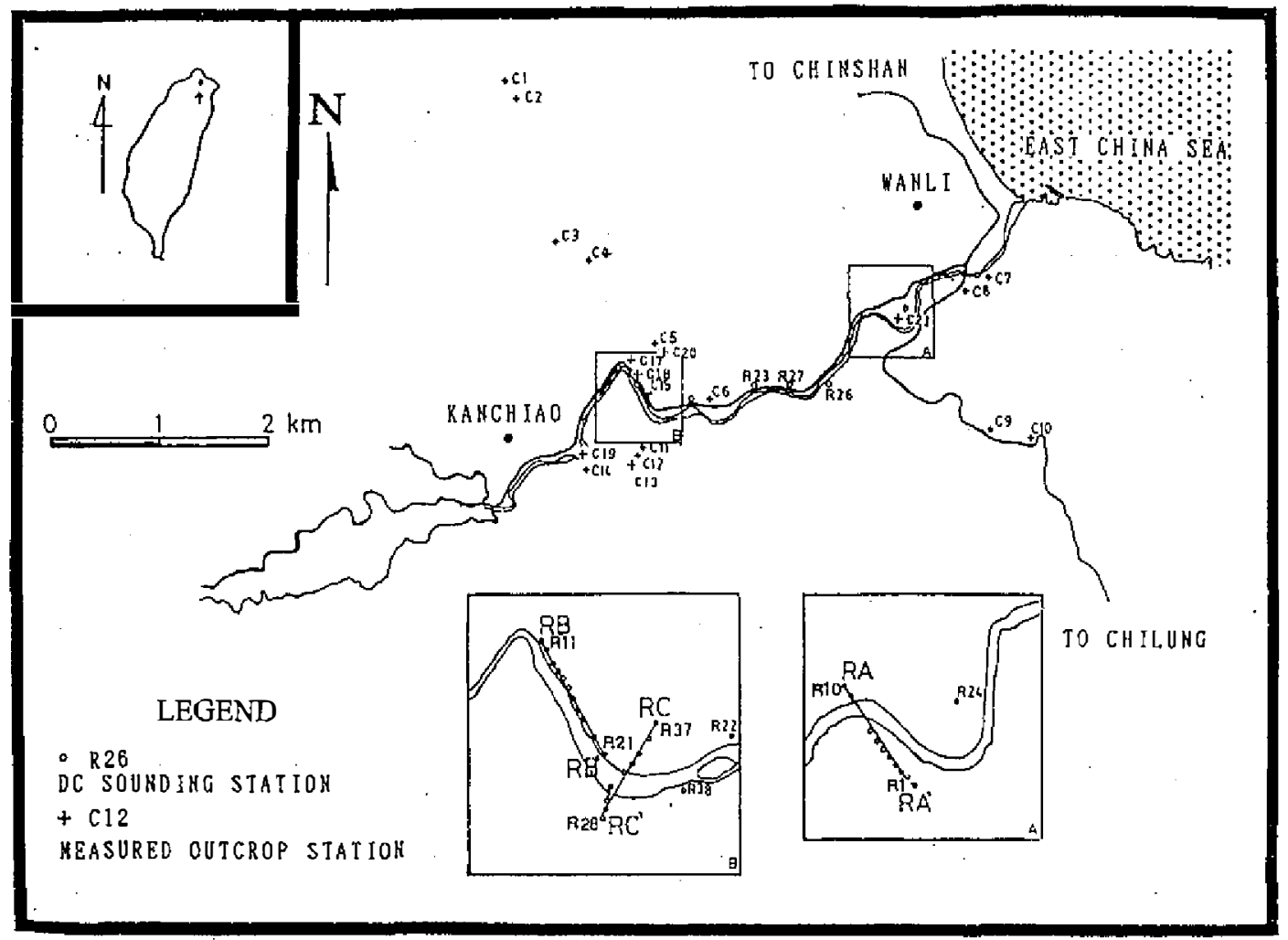

Fig. 6. The location map of DC sounding sites.

obtained. The finite element mesh used had 154 and 169 nodes for profiles RA - RA' and $\mathrm{RC}-\mathrm{RC}^{\prime}$ in the horizontal direction, and 20 and 19 nodes for profiles $\mathrm{RA}-\mathrm{RA}^{\prime}$ and $\mathrm{RC}-\mathrm{RC}^{\prime}$ in the vertical direction, respectively. The subsurface was divided into 47 blocks for each case. Figure 7a shows the final geoelectric model for profile RA - RA' .

Comparison is made between the synthetic (Fig. 7c) and the observed apparent resistivity pseudosections (Fig. 7b) for the RA - RA' profile. The two profiles are quite similar. The apparent resistivity sections show remarkable changes in resistivity values near the stations number 7 and 8 , which indicates that a possible fault crosses to the resistivity discontinuous zone. The interpreted position is near the expected location of the fault.

Figure $8 \mathrm{a}$ is an inverted final geoelectric model from the data shown in Fig. $8 \mathrm{~b}$ along the cross-section $\mathrm{RC}-\mathrm{RC}^{\prime}$. Figure $8 \mathrm{c}$ is the synthetic pseudosection of the geoelectric model shown in Fig. 8a. Comparison of Fig. 8c with the observed pseudosection (Fig. 8b) indicates that both figures are similar; the fault location has a clear expression in the resistivity discontinuous zone which is beneath the location labeled 34. Based on the local geology, an overthrust fault with great throws could be the cause of this resistivity discontinuous. The positions of the fault zone located at RA - RA' and RC- RC' were interpreted as shown in Figs. 7 and 8. 
(a)

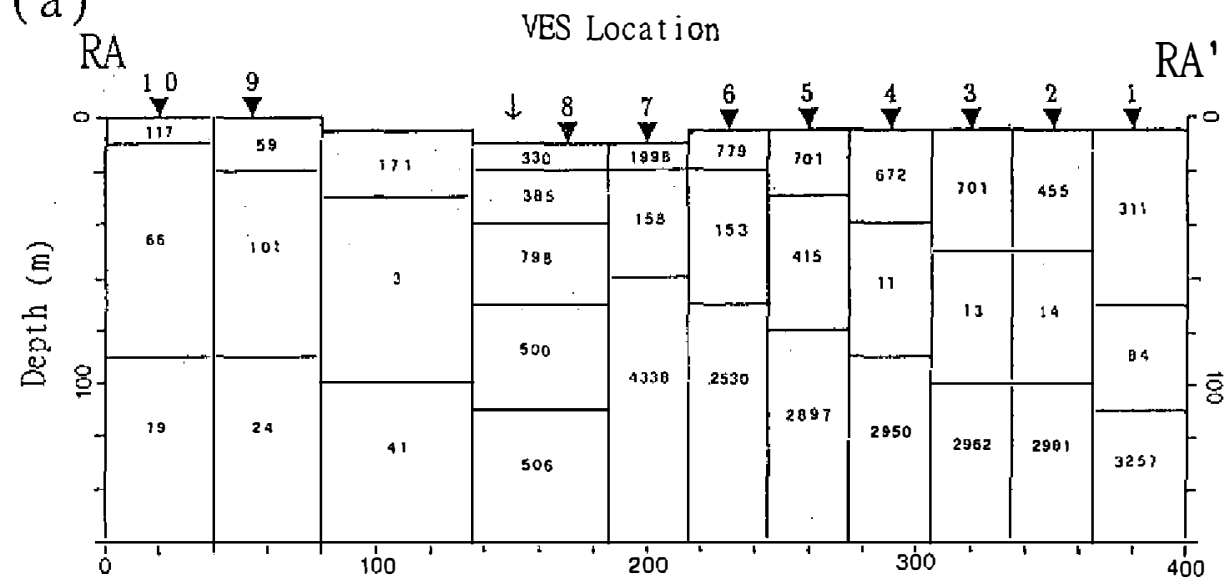

(b)

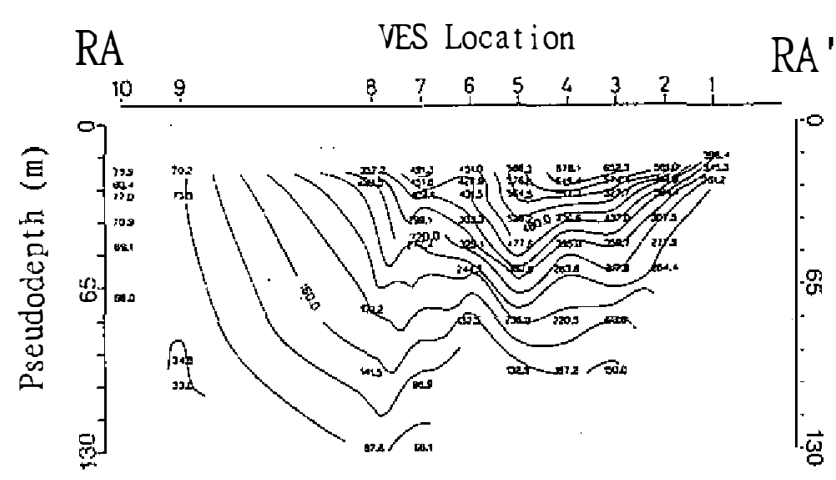

(c)

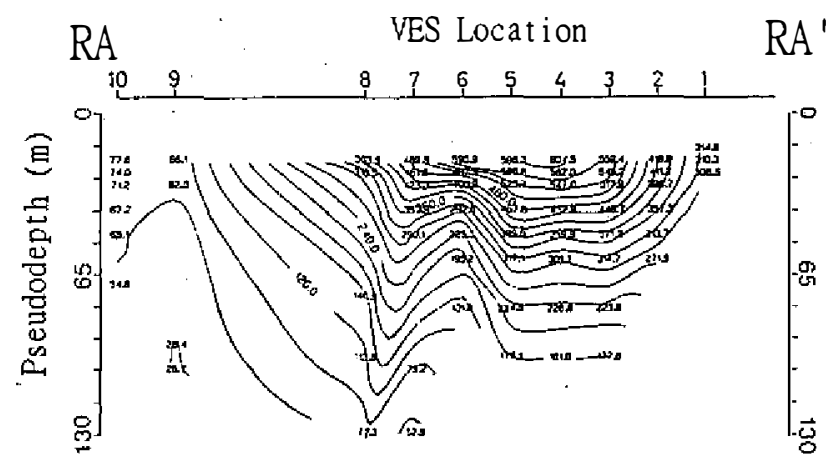

Fig. 7. (a) The final inversion model for the pseudosection shown in (b). (b) The apparent resistivity pseudosection along the traverse Line RA-RA' at Kanchiao test site. (c) The synthetic resistivity pseudosection for the inversion model shown in (b). 
(a)

VES Location

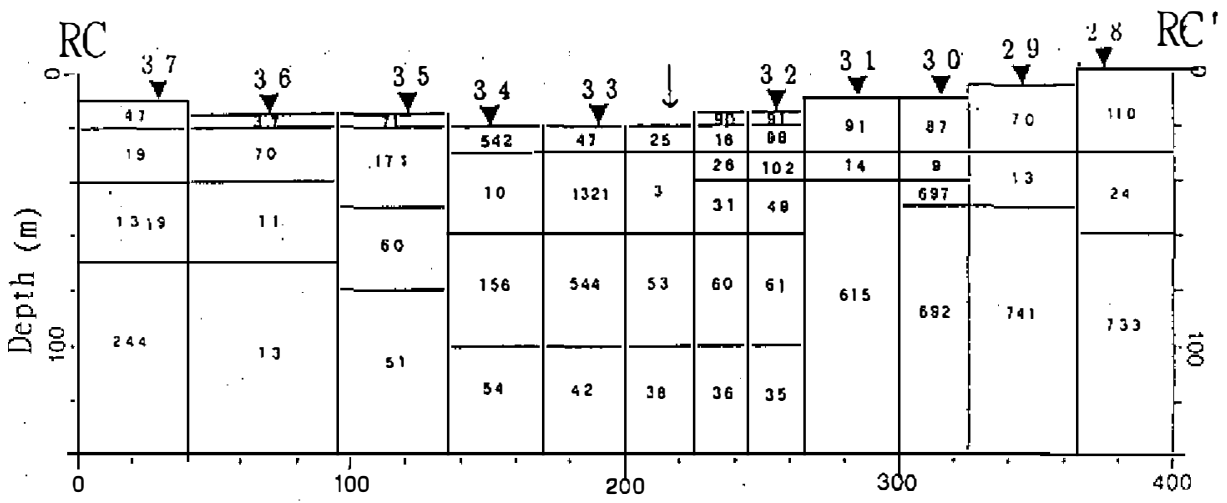

(b)

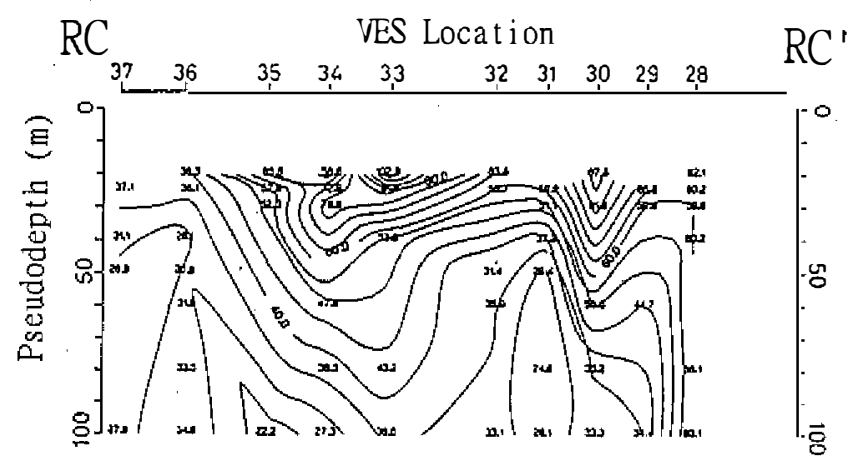

(c)

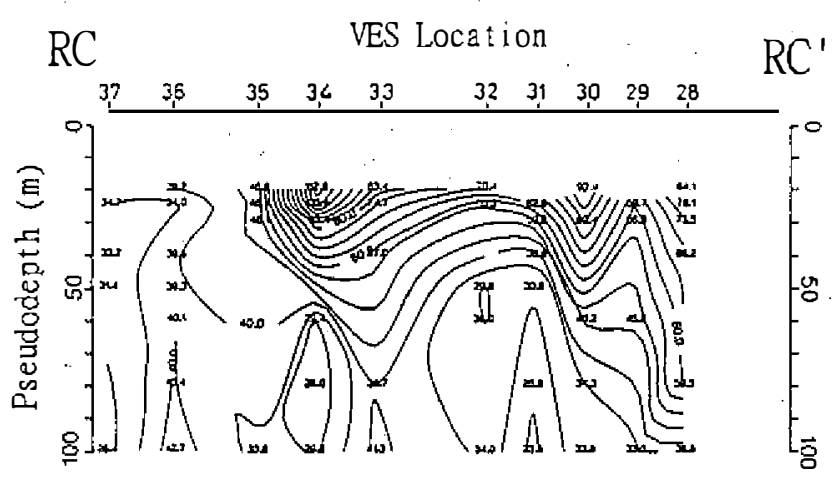

Fig. 8. (a) The final inversion model for the pseudosection shown in (b). (b) The apparent resistivity pseudosection of Line RC-RC' at Kanchiao test site. (c) The synthetic resistivity pseudosection for the inversion model shown in (b). 


\section{CONCLUSIONS}

This study reveals important facts concerning the special electrode configuration for direct current resistivity methods applied to detecting faults in a topographic area. From the above case studies, several points can be concluded: (1) Electric sounding with special arrays can obtain deeper subsurface information, especially in a topographic ground surface; (2) applying the scheme of incorporating the topography into a $2 \mathrm{D}$ finite element inversion to the sounding data can give a better subsurface picture than using a 1D inversion alone, since the algorithm is available to a high relief area with more complex subsurface geologic structures; and (3) the locations and characteristics of the Kanchiao fault can be identified by using a 2D DC resistivity surveys.

Acknowledgments Parts of this study were supported by the National Science Council grant (NSC78-0414-P008-03B), Republic of China. The authors thank the geoelectric group, National Central University, for their help to collect data in the field.

\section{REFERENCES}

Gloub, G. H., and C. Reinsch, 1970: Singular value decomposition and least-squares solutions. Num. Math., 14, 403-420.

Ho, C. S., M. Y. Hsu, and L. S. Jen, 1963: Fault structures in the Keelung coastal area, Taiwan. Proc. Geol. Soc. China, 6, 18-33.

Hsu, M. Y., 1967: The coal geology between Chinshan and Shimen, northern Taiwan. Bulletin of the Taiwan provincial Geological Survey, 19, 15-26. (in Chinese)

Huang, C. S., 1988: Geological map of Taiwan. Central Geological Survey, Sheet 4. (in Chinese)

Inman, J. R., 1975: Resistivity inversion with ridge regression. Geophysics, 40, 798-817.

Mooney, H. M.,E. Orellana, H. Pickett, and L. Ornheim, 1966: A resistivity computation method for layered earth models. Geophysics, 21, 192-203.

Oristaglio, M., and M. H. Worthington, 1980: Inversion of surface and borehole electromagnetic data for two-dimensional electrical conductivity models. Geophys. Prosp., 28, 633-657.

Rijo, L., W. H. Pelton, E. C. Feitosa, and S. H. Ward, 1977: Interpretation of apparent resistivity data from Apodi Valley, RGN, Brazil. Geophysics, 42, 483-506.

Tong, L. T., and C. H. Yang, 1990: Incorporation of topography into two-dimensional resistivity inversion. Geophysics, 55, 354-361.

Tripp, A. C., G. W. Hohmann, and C. M. Swift, 1984: Two-dimensional resistivity inversion. Geophysics, 49, 1708-1717.

Vozoff, K., and D. L.B. Jupp, 1975: Joint inversion of geophysical data. Geophys. J. Roy. Astr. Soc., 42, 977-991.

Yen, C. P., and P. Y. Chen, 1953: Geologic map of Taiwan (scale 1/50000), No. 9 (map of Taipei). Taiwan Provincial Geological Survey, 14p. (in Chinese) 
Zhdanov, M. S., and G. V. Keller, 1994: The geoelectrical methods in geophysical exploration. Elsevier, Amsterdam, 825-868. 